\title{
Evaluation of Vibration Signals Measured by 3-Axis MEMS Accelerometer on Human Face using Wavelet Transform and Classifications
}

\author{
Okan ORAL*, Suleyman BILGIN, Mehmet Umit AK
}

\begin{abstract}
Various studies have been conducted to reveal and analyse tissues from humans with distinct properties. The interpretation of human facial tissues was the subject of a few of these investigations. The aim of this study was to look at the energy ratios of vibration signals recorded from the human face using a 3-axis Micro-ElectroMechanic System accelerometer sensor. 9 various measurement points on the faces of the subjects used to receive the signals are then analysed using frequency characteristics. During the analysis process, wavelet transformation values are estimated and evaluated. Thus, these regions' frequency ranges can be calculated. In addition, critical properties extracted from signals of vibration using wavelet packet transformation analysis were used as inputs of classification methods. In the next step, multilayer perceptual neural networks (MLPNN) were evaluated. In addition, the support vector machine (SVM), decision tree (DT) and binary convolution neural networks (CNN) methods were evaluated, and the success rates were compared. Finally, it is seen that the energy ratios of the signals in the hard regions are low and the energy ratios of the signals in the soft regions are high. And it has been observed that higher accuracy rate is achieved with binary CNN than with other methods.
\end{abstract}

Keywords: Binary CNN; Multi-layer perceptron neural networks; Vibration signals; Wavelet packet transform

\section{INTRODUCTION}

The Micro-Electro-Mechanical Systems (MEMS) accelerometers are commonly used in the fields of medicine and biomechanics when diagnosing diseases [1]. MEMS is a technology which can be described in its most general form as miniaturized mechanical and electromechanical elements created using micro-manufacturing techniques [2]. Measurement with MEMS Accelerometers has been used over the past few decades as the instruments have low form factor, bio compatible materials and can therefore be easily attached to parts of the human body and acquire data from the respective body segments. The use of technology in such areas can provide high precision to predict the patient's symptoms and reduce the subjection of measurement and diagnosis to clinical evaluation alone, which depends on the diagnostic person and varies accordingly [3].

It is known that various studies have been done about tissues from humans showing various characteristics. In these investigations, vibration data have been used and solutions that can help detect diseases of different kinds are proposed. Coyte and colleagues [4] investigated wholebody vibration analysis and simulation techniques. This paper presents a review of the literature on a variety of subjects, including recent studies on modelling the biodynamic human sitting reaction to vibrations. Studies in which the sources of vibration explain the effects on different human tissues are also available in the literature. [5-8]. Vibration Energy Absorption was suggested by Dong and colleagues. This model has shown that different frequencies are effective on the finger, arm, and palm [9]. Balbinot et al. created a system to test human resonations integrated into the ZigBee network. On three axes, the built system allows for the separation of approximately $40 \mathrm{~Hz}$ human resonations. They showed that workers operating machines like tractors and diggers can experience back pain as a result of vibrations [10, 11]. Amar et al. [12] sought to clarify the human body's damping properties as a spring and damper mechanism against the application of vibrations to tissues from a human. The study's main goal is to find out more about to expose human tissues damping properties and the system's constants of spring and damping. In another related study [13] a method was developed estimating the damping properties of human tissues using a theoretical mass-damping spring model.

Analysis of the vibration data was used in the work on both the human face and body. The signals of vibrations that usually occur in the cords of the voice during conversation were the subject of these studies. [14-17]. These studies, on the other hand, use vibration data of different frequencies depending on the vocal cords. The vibration signals received by applying a constant frequency source of vibration to human tissues, on the other hand, may provide more detail [18]. In paranasal sinus radiological imaging methods such as direct $\mathrm{x}$-rays, ultrasonography, magnetic resonance imaging, computed tomography, are used [19]. The procedures used in those processes, however, produce harmful rays to the body of a human. Because of this, the aim of this study was to develop a technique that is more cost-effective without harming the human body with the MPU6050 sensor. [20]. There are some studies in the literature using accelerometers [21, 22]. Moreover, vibration signals measured using accelerometers have been found to assist in the diagnosis of disorders such as nasal polyps and sleep apnea $[14,16]$. It is assumed that in future studies the soft and hard interpretation of the tissues in the sinus regions can aid when it comes to determining the cause of nasal diseases.

This study developed a device that provides vibration excitation at a constant frequency using a vibration motor, a Micro-Electro-Mechanical Systems (MEMS) accelerometer sensor, and microcontroller-based hardware. Next, a total of 20 volunteer subjects were identified, and clinical tests were conducted to determine if they were suitable for the nasal cavity. The subjects' facial regions were stimulated by vibrations produced by the DC motor. MEMS was used to measure vibration data from 9 measurement points across the sinus regions. In this way, for all subjects a database has been developed, and their frequency characteristics were examined on a person's face for 9 points. Wavelet transformation methods were applied to vibration signals in the analysis phase. Bilgin et al. said 
that wavelet transform was an effective solution for the problem of decomposing Heart rate variability (HRV) signals into frequency band [23, 24]. Also, high accuracy has been achieved by using the CNN method in the classification of ECG signals [25]. Messner et al. extracted spectrogram features from lung sound recordings. And they compared different deep neural network architectures for binary classification and achieved high accuracy rate [26]. Cetin et al. analysed the dynamic walking signals of people with wavelet transform. They determined the energy intensity ratio of signals and the dominance rates of frequency bands [27].

In this research, frequency ranges of vibration data taken from measurement points were determined by using wavelet transform. In addition, critical properties extracted from vibration signals using wavelet packet transformation analysis were used as inputs of classification methods. Artificial neural networks (ANNs) are among common methods to classify real-time signals [28]. Multilayer perceptual neural networks (MLPNN), Support vector machine (SVM), Decision tree (DT) and Binary convolution neural network $(\mathrm{CNN})$ were used as classification methods and their accuracy rates were evaluated.

In this way, information about the dominant frequency bands of the regions on the face was obtained and the accuracy rates of these bands were determined. It aims to provide information about the frequency properties of living tissues.

\section{MATERIALS AND METHODS}

The acceleration sensor is one of the sensors that became very significant because of the advancement of MEMS technology. Accelerometers are sensors that transform acceleration from static movement or static gravity into analogue or digital electrical signals and produce significant output. They produce a voltage of electric output proportional to the oscillator value. Acceleration happens when there is a change in the speed of the target or in the speed the velocity is guided to. It also uses an acceleration sensor to measure variable states such as impact, vibration, rotation and slope [29]. A microcontroller-based MEMS vibration meter system (Fig. 1) was used in this research [30].

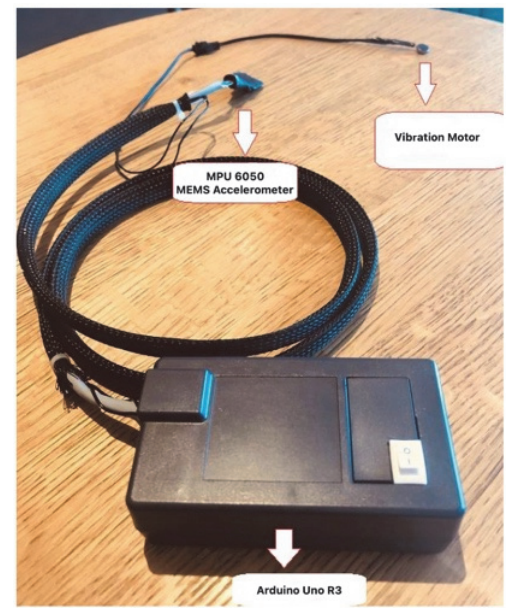

Figure $1 \mathrm{~A}$ microcontroller-based MEMS vibration meter system [30]
The system is made up of Arduino Uno R3 [31], MPU6050 3 - Axis MEMS accelerometer sensor [32], 10 $\times 3 \mathrm{~mm}$ DC vibration motor [33]. Vibration stimulation is applied to the face areas with a DC motor and vibration signals at a certain distance $(3 \mathrm{~cm})$ from the measurement points are recorded in the MPU6050 sensor. The MPU6050 sensor is a piezoelectric 3 - axis gyroscope sensor with 3 axis accelerometer and 6 - axis motion sensor. It also has a $250 \mathrm{~Hz}$ low-pass filter, which is switched on by default. The vibration signals are filtered before sampling with an anti-aliasing filter [34]. The sampling frequency of the vibration signals was determined to be $350 \mathrm{~Hz}$ in this sample. The operating range of vibration motor was 20 $200 \mathrm{~Hz}$ [4, 34]. An oscilloscope was used to set the frequency of DC motor to $160 \mathrm{~Hz}$ in the Akdeniz University Faculty of Engineering laboratory.

\subsection{Database Acquisition}

The database was created with vibration signals from 20 healthy volunteers. The examination of whether the volunteers are healthy or not was carried out at Akdeniz University Medical Faculty Hospital (Fig. 2).

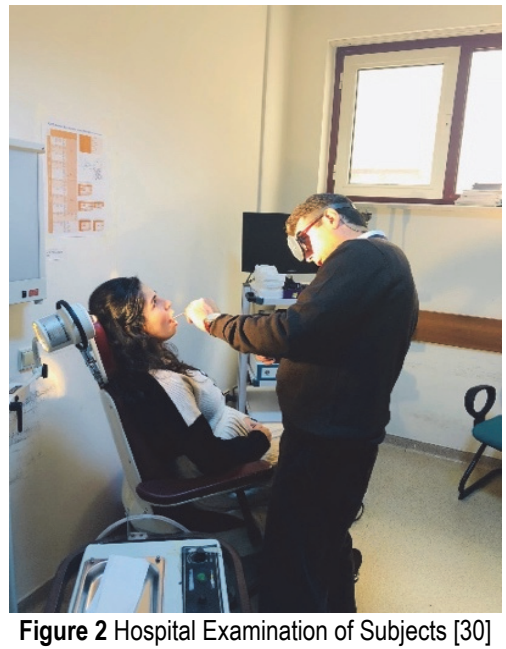

Some information about the volunteers is as follows: There are 12 male and 8 female volunteers with an age range of $25-35$. The male subjects participating in the study have a height range of $1.68-1.86 \mathrm{~m}$, a body weight of $65-105 \mathrm{~kg}$, and a body mass index range of 20.52 30.82 . The female subjects participating in the study have a height range of $1.58-1.80 \mathrm{~m}$, a weight range of $53-75$ $\mathrm{kg}$, and a body mass index range of $19.00-26.89$.

During the measurement, the participants were asked to keep their heads still and not to wear glasses. Additionally, $15 \mathrm{~Hz}$ High Pass Filter was used to eliminate the head movements during data recording. There were no glasses found affecting the acquisition of data in facial areas. A database was created by recording the vibration data from the regions determined on the percentage.

\subsection{Measurement Regions}

Measurement points were created for the regions $\mathrm{F}$ (Forehead Area), MR (Right-Cheek Area) and ML (LeftCheek Area) [33]. These points consist of 9 points in the frontal and maxillary sinus regions. There is a $3 \mathrm{~cm}$ 
distance between each measurement point vibration motor. Measurements points are shown in Fig. 3. F1, F2, F3 are located near the frontal sinus over the brow; MR1, MR2, MR3 are located near the right cheek maxillary sinuses; and ML1, ML2, ML3 are located near the left cheek maxillary sinuses $[35,36]$. In addition, where the vibration motor is used, those points are displayed in black circles.

MPU6050 accelerometer sensor was mounted separately on F1, F2, F3, MR1, MR2, MR3, ML1, ML2, ML3 points. Throughout the measurement, the vibration motor was fixed on the body surface, indicated by the black circle. For example, the area indicated by the black circle in the $\mathrm{F}$ region was positioned by a vibration motor and measurements were taken separately from points F1-F2F3. The same procedure was repeated for MR and ML regions in the same way.

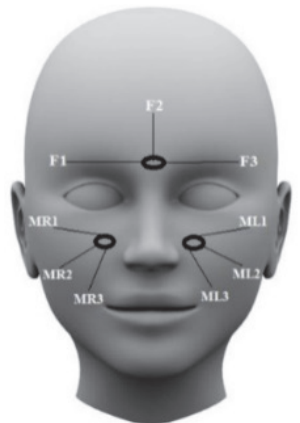

Figure 3 Vibration Motor and Measurement Points [34]

The vibration data from the measurement points was separated and passed to the device as $x-y-z$ values using an Arduino Uno R3 and Arduino program. The collected vibration data's $x-y-z$ values were analysed and interpreted. For all measurements, $z$ values are identical, for this reason only $x-y$ values were considered. The combination of $x-y$ values was then taken, and the sum of change with the combination was observed. The $z$ axis of the MPU-6050 sensor was kept constant during all measurements, and measurements were taken at the same place. Fig. 4 depicts the $x-y-z$ axes in relation to the measurement points on the MPU-6050's face.

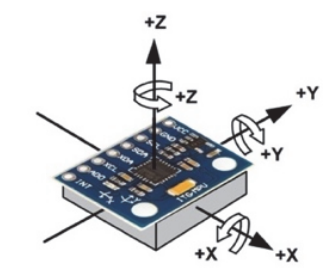

Figure 4 MPU-6050 $x-y-z$ axis [31]

Then wavelet transform method was used to evaluate vibration data. The general block diagram of the system is shown in Fig. 5 [37].

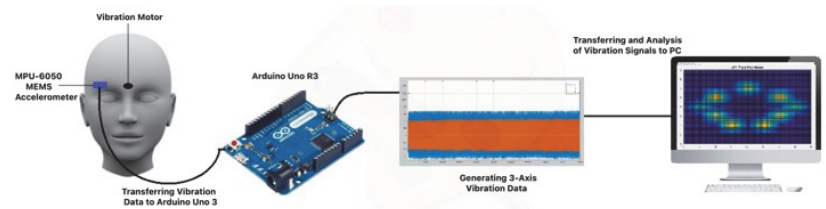

Figure 5 System Block Diagram [30]
The vibration data was then evaluated using the wavelet transform process [37].

According to the system flow diagram shown in Fig. 6 , a sensor must be placed on the face in order to detect the vibration signal first. In order to measure the vibration signal, MPU-6050 MEMs (capable of measuring in the $x$ $y-z$ direction) accelerometer sensor is used in the circuit. Arduino Uno R3 was used to obtain the data and send it to the computer environment. Arduino Uno R3 board is a microcontroller module that can provide this communication with serial communication protocols and ADC modules.

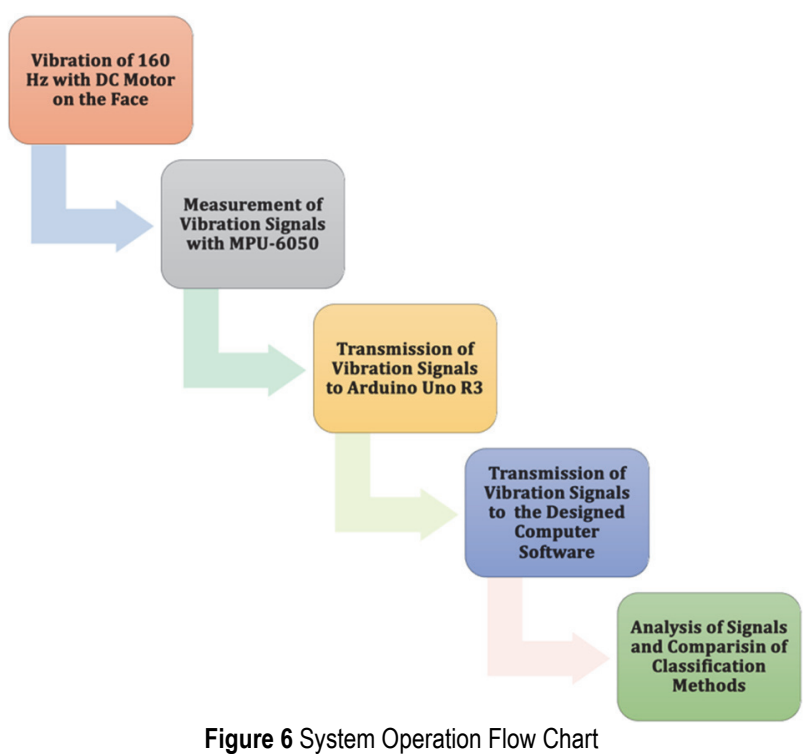

The vibration signals obtained were transferred to the computer environment via the USB protocol via the Arduino Uno R3 board. Numerical data were recorded with the graphical interface software created in the Matlab program on the computer and displayed on the screen according to the time history area. Vibration signals received were analysed according to signal processing methods and detection and classification algorithms through the software developed within the scope of the study. The characteristics of the vibration signals received on the human face were created and interpreted. By performing time, frequency, time-frequency separations of vibration signals, features are extracted and separated into lower frequency bands.

\subsection{Wavelet Packet Transform (WPT)}

Wavelet Packet Transform (WPT) is a discrete decomposition type of Wavelet Transform (WT) [38]. The approximation and definition coefficients at each stage are further decomposed in WPT signal decomposition. In DWT, description coefficients are passed down to the next point, unchanged. Both the coefficients in WPT are therefore decomposed in each stage [24, 38]. WPT function is described; Packet Transform (WPT) is a type of Wavelet Transform (WT) using a discrete decomposition [38].

$W_{m, j, n}(t)=2^{-\frac{m}{2}} W_{j}\left(2^{-m} t-n\right)$ 
' $j$ ' means node index at every $m$ level. The WP tree of decomposition for $W_{i, j}$ and decomposition for a single node is shown in the figure below (Fig. 7).

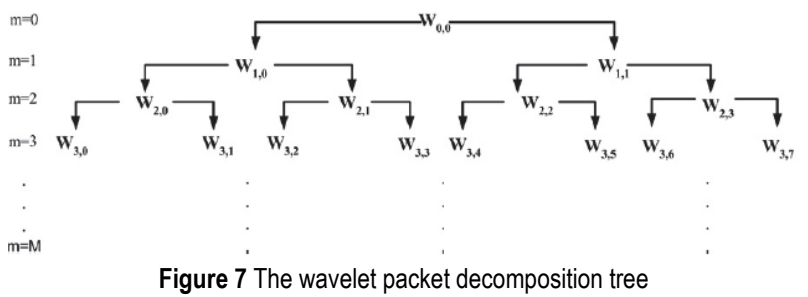

The wavelet packages and frequency ranges examined are shown in Tab. 1. Also, $350 \mathrm{~Hz}$ sampling frequency was used.

Table 1 Wavelet packages and frequency ranges

\begin{tabular}{|c|c|c|}
\hline$m$ & Wavelet Level & Frequency Range $/ \mathrm{Hz}$ \\
\hline 0 & $W_{0,0}$ & $0-175 \mathrm{~Hz}$ \\
\hline 1 & $W_{1,1}$ & $87.5-175 \mathrm{~Hz}$ \\
\hline 2 & $W_{2,2}$ & $87.5-131.25 \mathrm{~Hz}$ \\
\hline 3 & $W_{3,5}$ & $109.375-131.25 \mathrm{~Hz}$ \\
\hline \multirow{2}{*}{4} & $W_{4,10}$ & $109.375-120.3125 \mathrm{~Hz}$ \\
\cline { 2 - 3 } & $W_{4,11}$ & $120.3125-131.25 \mathrm{~Hz}$ \\
\hline
\end{tabular}

The total energy ratios of the signals are used to calculate the detail and approximation coefficients. The $E_{C}$ is the energy of the examined vibration signal. $E_{T}$ is the total energy of the vibration signals and $E_{P}$ is percentage of energy ratio.

$$
E_{P}=\frac{E_{C}}{E_{T}} \cdot 100
$$

\subsection{Classification Methods}

In this study, more comprehensive classification methods are needed to differentiate soft and hard tissues using calculated energy values. Although there are many classification methods in the literature, one of the most used methods is ANN [28]. MLPNN, SVM, DT and binary CNN structures for classification were also evaluated during the study.

MLPNN is a type of ANN algorithm consisting of two or more neuronal layers within each layer. The MLPNN structure is based on a perceptron model fundamentally [28]. Mathematically a perceptron model in the layer $j_{t h}$ is determined as:

$$
y=f\left(\sum_{i=0}^{N} x_{i} w_{i j}+\theta_{j}\right),(j=1,2, \ldots, M)
$$

where $N$ refers to the number of neurons in the input layer, $M$ is the number of layers, $x_{i}$ is the $i_{t h}$ neuron in a hidden layer, $w_{i}$ is weights for each input, $\theta_{j}$ is the bias of the perceptron, $f($.) is the activation function, and $y$ refers to the output of the perceptron in the $j_{t h}$ layer.

SVM method is a controlled learning algorithm recommended for solving classification and regression problems. It solves any classification or regression problem by transforming it into a second-order programming problem without interfering with local solutions. It also can make high generalizations. The output signal $y$ of SVM network is shown as the function of variable $x$ :

$$
y=\sum_{i} w_{i} k\left(s_{i}, x\right)+b
$$

where $s_{i}$ parameters are the support vectors, $w_{i}$ parameters are the weights, $b$ is the bias, and $k$ is a kernel function [28]. The size of the kernel (KS) is computed between 0.2 and 3.5 , using a sub-sampling heuristic method.

DT makes use of a multi-stage or sequential approach to the classification process. The basic principle of the DT structure is to obtain results by splitting the data into small pieces in the shortest possible time. DT is one of the most used methods among the machine learning methods, which have been used in particular since the early times in classification. The tree must be properly built in order to achieve high precision in the DT process [39]. DT is comprised of roots, branches and leaves and, in terms of form, resembles a tree. DT starts with a root node and separates a large number of datasets into small groups and splits them into branches as they go down. Joints on the tree are referred to as nodes. Fig. 8 shows the working systematic of the DT. The internal nodes in the DT represent the property, the branch represents the rule of decision and the leaf represents the results [40].

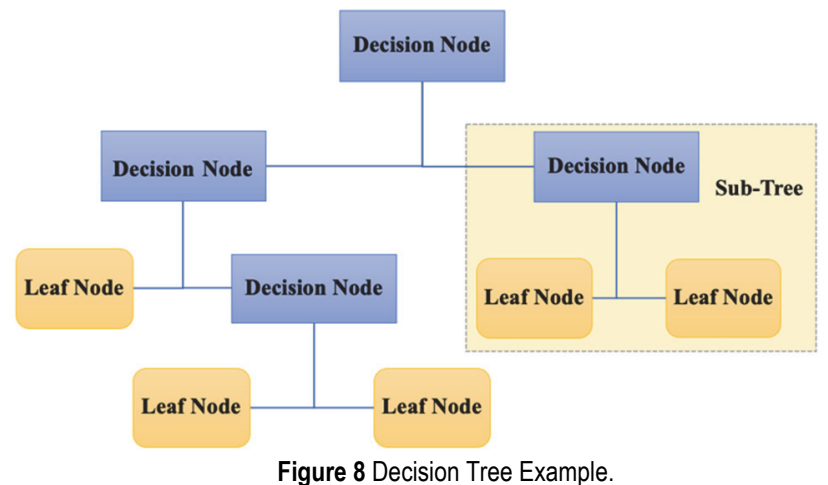

$\mathrm{CNN}$ is made up of a variety of different types of layers that operate in order, with the next layer's input being the output of the previous layer. The input/output of one layer is called "function map," and the parameters of another layer are called "weights." One or more Fully-ConnectedLayers adopt cascaded convolutionary layers in a typical $\mathrm{CNN}$ structure. In this study, binary CNN were used in the processing of forward propagation using binary weights and data [41]. In binary convolutional neural networks, weights and activations are constrained to a single value +1 or -1 . The deterministic binarization function is described as follows [42].

$x^{b}=\left\{\begin{array}{l}+1, x^{r} \geq 0 \\ -1, x^{r}<0\end{array}\right.$

where $x^{b}$ is the binarized variable, and $x^{r}$ is the real-valued variable. During the backpropagation, the StraightThrough Estimator (STE) method 5 is adapted to calculate the derivatives of the binarization functions as follows, where $C$ is the loss function 6 . 
$\frac{\partial C}{\partial x^{r}}=\frac{\partial C}{\partial x^{b}}$

Rotation estimation is also known as cross-validation. It is a way for determining how a statistical analysis' conclusions would generalize to a new data set. To begin, the information is divided into $k$ equal or nearly equal segments or folds. Training and testing on these partitioned folds is done in $\mathrm{k}$ iterations, with each iteration leaving one fold for testing and training the model on the remaining $k$ -1 folds. The model accuracy is calculated by averaging the accuracy attained in each iteration. It is worth noting that data is frequently stratified before being divided into $\mathrm{k}$ pieces. Stratification is the process of organizing data so that each fold accurately represents the entire [43].

In the study, properties obtained with WPT were first applied to MLPNN inputs. The number of layers and neurons has been tried to the point where the accuracy rate is maximum. Also, three-fold cross-validation (TFCV) was used for evaluation and validation of the classifications. TFCV is a cross-validation method considering the database partitioning rules in ANN methods. In addition to MLPNN, SVM, DT and binary CNN classification methods were also applied, and success rates were evaluated.

\section{RESULT}

In this research, vibration data were recorded with the designed microcontroller-based vibration meter device. These vibration data were obtained using the computer interface that was created, and a database was obtained. The vibration signals from 9 measurement points identified in the facial regions of 20 healthy subjects are stored in the database. The energy ratio analysis of the F, MR, ML regions was performed using WPT and the vibration signals were analysed using WPT. Finally, the properties derived from WPT analysis were evaluated with classification methods.

Firstly, energy ratio analysis of F, MR, ML regions in $W_{3,5}$ is demonstrated in Fig. 9. The frequency range of this package is $109.375-131.25 \mathrm{~Hz}$, and when the energy ratios of the $\mathrm{F}, \mathrm{MR}, \mathrm{ML}$ regions are examined, the region with the lowest energy ratio $\left(E_{P}\right)$ is $\mathrm{F}$ region and the highest energy ratio $\left(E_{P}\right)$ is $\mathrm{ML}$ region.

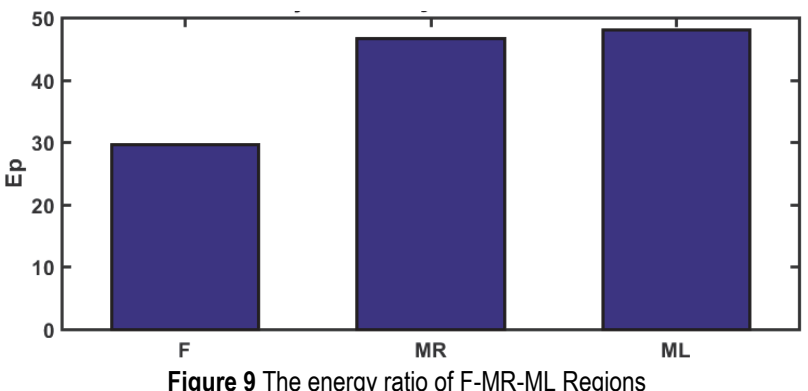

Also, energy ratio analysis of F1-F2-F3-MR1-MR2MR3-ML1-ML2-ML3 regions in $W_{3,5}$ is shown in Fig. 10. When the energy ratios of the F1-F2-F3-MR1-MR2-MR3ML1-ML2-ML3 regions are examined, the region with the lowest energy ratio $\left(E_{P}\right)$ is $\mathrm{F} 2$ region and the highest energy ratio $\left(E_{P}\right)$ is ML3 region.

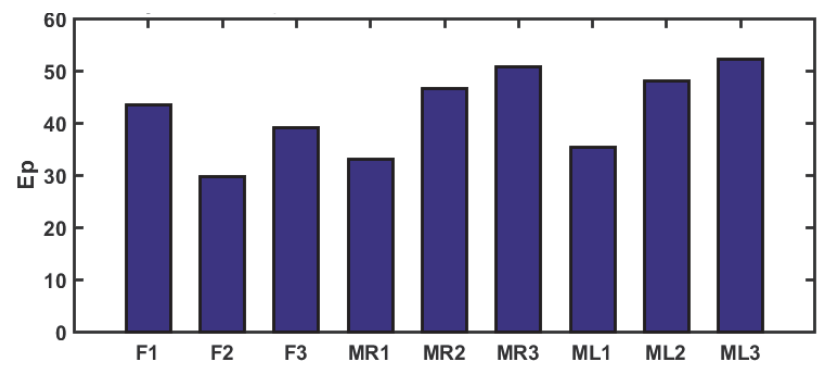

Figure 10 The energy ratio of the F1-F2-F3-MR1-MR2-MR3-ML1-ML2-ML3

When the vibration signals received from all subjects were examined, it was observed that the energy rates of the signals received from the $\mathrm{F}$ region were the lowest and the energy rates of the signals received from the ML region were the highest. Similarly, it was observed that the energy rates of the signals received from the F2 points were the lowest and the energy rates of the signals received from the ML3 points were the highest.

The energy ratios of the different frequency ranges obtained with WPT are applied to MLPNN inputs and their accuracy percentages are evaluated. The results of this analysis are shown in Tab. 2. It belongs to the MLPNN structure, which has three neurons in the input layer, two hidden layers with ten neurons, and one neuron in the output layer. The number of layers and neurons is determined by their classification success. The activation function is selected as the tangent sigmoid in each neuron in all structures for performance. Energy ratios in all wavelet packages were applied to the input layer and evaluated. Measured softness hardness interpretation was used as the output value.

Table 2 The comparison results of MLPNN

\begin{tabular}{|c|c|c|}
\hline Wavelet Packet & Frequency Range / Hz & Accuracy Rate / \% \\
\hline$W_{4,1}, W_{4,2}, W_{4,3}$ & $(0-32.8125)$ & 65.74 \\
\hline$W_{4,2}, W_{4,3}, W_{4,4}$ & $(10.9375-43.75)$ & 66.48 \\
\hline$W_{4,3}, W_{4,4}, W_{4,5}$ & $(21.875-54.6875)$ & 66.66 \\
\hline$W_{4,4}, W_{4,5}, W_{4,6}$ & $(32.8175-65.625)$ & 66.29 \\
\hline$W_{4,5}, W_{4,6}, W_{4,7}$ & $(43.75-76.5625)$ & 66.66 \\
\hline$W_{4,6}, W_{4,7}, W_{4,8}$ & $(54.6875-87.5)$ & 68.33 \\
\hline$W_{4,7}, W_{4,8}, W_{4,9}$ & $(87.5-120.3125)$ & 72.70 \\
\hline$W_{4,8}, W_{4,9}, W_{4,10}$ & $(98.4375-131.25)$ & 75.00 \\
\hline$W_{4,9}, W_{4,10}, W_{4,11}$ & $(109.375-131.25)$ & 77.22 \\
\hline$W_{4,10}, W_{4,11}, W_{4,12}$ & $(120.3125-153.125)$ & 76.11 \\
\hline$W_{4,11}, W_{4,12}, W_{4,13}$ & $(131.25-164.0625)$ & 69.44 \\
\hline$W_{4,12}, W_{4,13}, W_{4,14}$ & $(142.1875-175)$ & 69.25 \\
\hline
\end{tabular}

The highest accuracy rate was determined in packages in the frequency range of $109.375-131.25 \mathrm{~Hz}$. The accuracy percentages are calculated as $77.22 \%$ in MLPNN.

The percentages of accuracy were calculated in the final part of the analysis by applying the energy ratios of different frequency ranges obtained with WPT to SVM, DT and binary CNN inputs. Accuracy percentages for these methods were as follows; $74.20 \%$ in SVM, $72.80 \%$ in DT and $79.62 \%$ in binary CNN. Therefore, according to the comparison of the results, it is seen that binary CNN is the most powerful classification method.

Several studies are available in the literature on the investigation of vibration signals [44, 45]. MEMS technology is said to be able to provide information on tissue density [46]. Vibration data were also used to diagnose disorders such as sleep apnea and nasal polyps [14, 16, 35, 47, 48]. In contrast to previous research, vibration data obtained by applying a constant frequency 
source of vibration to human faces was calculated, and the energy ratio distribution of these vibration signals was determined. These energy rates were analysed with MLPNN, SVM, DT, binary CNN and accuracy rates were determined.

\section{CONCLUSION}

In this research, vibration signals were recorded on the human face with a microcontroller-based vibration meter device and transferred to the computer. These data were registered using the computer interface that was created, and a database was created. Then the vibration data were analysed with WPT and the energy ratios of these signals were compared. Finally, the results from WPT analysis have been validated with MLPNN, SVM, DT and binary $\mathrm{CNN}$. Through the analysis of the results, binary CNN is seen as the most efficient method of classification.

The results gave information about the energy ratios of the vibration signals received from the F-MR-ML regions of this model. When these values are examined, it can be seen that different points on the face give different answers against a fixed source of vibration. The results show that this model is capable of distinguishing between hard and soft separations in these facial areas. In future research, it is hoped that this model can help diagnose diseases of nasal cavity origin. As a result, it aims to propose a more environmentally friendly and cost-effective process.

\section{Acknowledgements}

This study was supported by Akdeniz University Scientific Research Projects Coordination Unit with project FBA-2019-4404. In addition, with the 2012KAEK-20 decision, Akdeniz University Faculty of Medicine Clinical Research Ethics Committee has obtained permission to conduct the study in terms of scientific and ethical issues. We would like to thank Prof. Dr. Alper Tunga DERIN, Prof. Dr. Narin DERIN and Assist. Prof. Dr. Hamza Feza CARLAK for their valuable contribution to the study.

\section{REFERENCES}

[1] Nez, A., Fradet, L., Laguillaumie, P., Monnet, T., \& Lacouture, P. (2016). Comparison of calibration methods for accelerometers used in human motion analysis. Medical Engineering \& Physics, 38(11), 1289-1299. https://doi.org/10.1016/j.medengphy.2016.08.004

[2] Sumbul, H. \& Yuzer, A. H. (2016). 3D Monitoring of Lying Position for patients with positional sleep apnea syndrome. Journal of New Results in Science, 5, 59-70. https://dergipark.org.tr/en/pub/jnrs/issue/27333/287730

[3] Preeti, M., Guha, K., Baishnab, K. L., Dusarlapudi, K., \& Raju, K. N. (2019). Low frequency MEMS accelerometers in health monitoring-A review based on material and design aspects. Materials Today: Proceedings, 18(2019), 21522157. https://doi.org/10.1016/j.matpr.2019.06.658

[4] Coyte, J. L., Stirling, D., Du, H., \& Ros, M. (2015). Seated whole-body vibration analysis, technologies, and modeling: a survey. IEEE Transactions on Systems, Man, and Cybernetics: Systems, 46(6), 725-739. https://doi.org/10.1109/TSMC.2015.2458964
[5] Peter, H., Podszus, T., \& Wichert, P. (2013). Sleep related disorders and internal diseases. Springer Science and Business Media.

[6] Zhi-Fei, Z., Zhong, X., \& Yansong, H. (2009). Design of measurement and evaluation system for human exposure to mechanical vibration. In2009 9th International Conference on Electronic Measurement \& Instruments, 504-508. https://doi.org/10.1109/ICEMI.2009.5274819

[7] Yanxi, R. \& Qingxia, L. (2010, June). Implementation of human vibration test and evaluation system based on virtual instrument. 2010 International Conference on Mechanic Automation and Control Engineering, 2430-2435. https://doi.org/10.1109/MACE.2010.5535864

[8] Kitamura, T. (2012). Measurement of vibration velocity pattern of facial surface during phonation using scanning vibrometer. Acoustical Science and Technology, 33(2), 126128. https://doi.org/10.1250/ast.33.126

[9] Dong, R. G., Schopper, A. W., McDowell, T. W., Welcome, D. E., Wu, J. Z., Smutz, W. P., \& Rakheja, S. (2004). Vibration energy absorption (VEA) in human fingers-handarm system. Medical Engineering \& Physics, 26(6), 483492. https://doi.org/10.1016/j.medengphy.2004.02.003

[10] Balbinot, A., Chiaramonte, M. S., \& Koenig, D. (2008). Zigbee network for measurement of human vibration. 11th IEEE International Conference on Computational Science and Engineering Workshops, IEEE, 8-12. https://doi.org/10.1109/CSEW.2008.18

[11] De Capua, C., Meduri, A., \& Morello, R. (2009). A handarm vibration meter monitoring the percussion exposure for health risk prevention applications. 2009International Workshop Medical Measurements and Applications, IEEE, 45-50. https://doi.org/10.1109/MEMEA.2009.5167952

[12] Amar, M. R. (2010). Estimation of mechanical properties of soft tissue subjected to dynamic impact. Industrial and Management Systems Engineering, University of Nebraska, Lincoln.

[13] Alkhaledi, K. (2010). Human response to soft tissue impact. Industrial and Management Systems Engineering, University of Nebraska, Lincoln.

[14] Morillo, D. S., Ojeda, J. L. R., Foix, L. F. C., \& Jiménez, A. L. (2010). An accelerometer-based device for sleep apnea screening. IEEE Transactions On Information Technology In Biomedicine, 14(2), 491-499. https://doi.org/10.1109/TITB.2009.2027231

[15] Chen, F. C., Ma, E. P. M., \& Yiu, E. M. L. (2014). Facial bone vibration in resonant voice production. Journal of Voice, 28(5), 596-602. https://doi.org/10.1016/j.jvoice.2013.12.014

[16] Orun, E. (2017). Analysis of the fundamental frequency and nasal sound in patients with nasal polyps. Institute of Science, Istanbul University.

[17] Kitamura, T., Hatano, H., Saitou, T., Shimokura, Y., Haneishi, E., \& Kishimoto, H., (2013). Pilot study of vibration pattern measurement for facial surface during singing by using scanning vibrometer. In Proceedings of Stockholm Music Acoustics Conference, 275-278.

[18] Torvinen, S., Kannus, P., SievaĖnen, H., JaĖrvinen, T. A., Pasanen, M., Kontulainen, S., \& Vuori, I., (2002). Effect of a vibration exposure on muscular performance and body balance. Clinical Physiology and Functional Imaging, 22(2), 145-152. https://doi.org/10.1046/j.1365-2281.2002.00410.x

[19] Ak M. U. (2019). Investigation the frequency characteristics of vibration signals on the human face. Institute of Science, Akdeniz University.

[20] Stemme, G. (2007). Medical technology MEMS. IET Seminar on Micro Electro-Mechanical Systems, 1-24. https://doi.org/10.1049/ic.2007.1704

[21] Jansi, R. \& Amutha, R. (2020). Detection of fall for the elderly in an indoor environment using a tri-axial 
accelerometer and kinectdepth data. Multidimensional Systems and Signal Processing, 31(4), 1207-1225. https://doi.org/10.1007/s11045-020-00705-4

[22] Chakraborty, S., Chattaraj, S., \& Mukherjee, A. (2018). Performance evaluation of particle filter resampling techniques for improved estimation of misalignment and trajectory deviation. Multidimensional Systems and Signal Processing, 29(3), 821-838. https://doi.org/10.1007/s11045-017-0472-1

[23] Addison, P. S. (2017). The illustrated wavelet transform handbook: introductory theory and applications in science, engineering, medicine and finance. CRC Press.

[24] Bilgin, S., Çolak, O. H., Polat, O., \& Koklukaya, E. (2009). Estimation and evaluation of sub-bands on LF and HF basebands in HRV for Ventricular Tachyarrhythmia patients. Expert Systems with Applications, 36(6), 10078-10084. https://doi.org/10.1016/j.eswa.2009.01.014

[25] Baloglu, U. B., Talo, M., Yildirim, O., San Tan, R., \& Acharya, U. R. (2019). Classification of myocardial infarction with multi-lead ECG signals and deep CNN Pattern Recognition Letters, 122, 23-30. https://doi.org/10.1016/j.patrec.2019.02.016

[26] Messner, E., Fediuk, M., Swatek, P., Scheidl, S., SmolleJüttner, F. M., Olschewski, H., \& Pernkopf, F. (2020). Multichannel lung sound classification with convolutional recurrent neural networks. Computers in Biology and Medicine, 122, 103831 . https://doi.org/10.1016/j.compbiomed.2020.103831

[27] Cetin, E., Bilgin, S., \& Oral, O. (2019). A Walking and balance analysis based on pedobarography. In the International Conference on Artificial Intelligence and Applied Mathematics in EngineeringSpringer, 906-913. https://doi.org/10.1007/978-3-030-36178-5_80

[28] Bilgin, S. \& Akin, Z. E. (2018). Gait pattern discrimination of ALS patients using classification methods. Turkish Journal of Electrical Engineering \& Computer Sciences, 26(3), 1367-1377. https://doi.org/10.3906/elk-1708-221

[29] Oral, O., Colak, O., \& Bayhan, M. N. G. (2019). Monitoring of tool vibration in the milling of Ti6Al4V with MEMS accelerometer. The European Journal of Science and Technology, 17, 1134-1144. https://doi.org/10.31590/ejosat.654256

[30] Ak, M. Ü., Bilgin, S., Oral, O., Cetin, E., Carlak, H. F., Derin, A. T., \& Derin, N. (2019). A new system design for measuring vibration effect in live tissues. Medical Technologies Congress, 1-4. https://doi.org/10.1109/TIPTEKNO.2019.8895210

[31] Arduino Genuino UNO, https://www.arduino.cc/en/Main/ArduinoBoardUno.

[32] MPU-6000 and MPU-6050 Product Specification, Retrieved from https://invensense.tdk.com/wp-content/uploads/ 2015/02/MPU-6000-Datasheet1.pdf

[33] Vibration motor datasheet, Retrieved fromhttps://www.precisionmicrodrives.com/product/datash eet/310-103-10mm-vibration-motor-3mm-typedatasheet.pdf.

[34] Ak, M. U., Bilgin, S., Oral, O., Çetin, E., Carlak, H. F., Derin, A. T., \& Derin, N. (2020). Evaluation of vibration measurements on human face using median and maximum frequencies. IET Science, Measurement \& Technology. https://doi.org/10.1049/iet-smt.2019.0381

[35] Fokkens, W. J., Lund, V. J., Mullol, J., Bachert, C., Alobid, I., Baroody, F., Cohen, N., Cervin, A., Douglas, R., Gevaert, P., Georgalas, C., Goossens, H., Harvey, R., Helling, P., Hopkins, C., Jones, N., Joos, G., Kalogjera, L., Kern, B., Kowalski, M., Price, D., Riechelman, H., Schlosser, R., Brent, S., Thomas, M., Toskala, E., Voagels, R., Wang, Y. D., \& Wormald, P. J. (2012). European position paper on rhinosinusitis and nasal polyps. A Summary for Otorhinolaryngologists Rhinology, 50(1), 1-12. https://doi.org/10.4193/rhino12.000

[36] Cler, M. J., Lien, Y. A. S., Braden, M. N., Mittelman, T., Downing, K., \& Stepp, C. E. (2016). Objective measure of nasal air emission using nasal accelerometry. Journal of Speech, Language and Hearing Research, 59(5), 1018-1024. https://doi.org/10.1044/2016 JSLHR-S-15-0407

[37] Ak, M. U., Bilgin, S., Oral, O., Çetin, E., Carlak, H. F., Derin, A. T., \& Derin, N. A. (2019). Median and maximum frequency analysis of vibration signals on human face, International Conference on Research in Engineering and Technology.

[38] Bilgin, S., Colak, O. H., Koklukaya, E., \& Ari, N. (2008). Efficient solution for frequency band decomposition problem using wavelet packet in HRV. Digital Signal Processing, 18(6), 892-899. https://doi.org/10.1016/j.dsp.2008.04.007

[39] Cakir, M., Guvenc, M. A., \& Mistikoglu, S. (2021). The experimental application of popular machine learning algorithms on predictive maintenance and the design of IIoT based condition monitoring system. Computers \& Industrial Engineering, 151(106948), 1-14. https://doi.org/10.1016/j.cie.2020.106948

[40] Salin, E. D. \& Winston, P. H. (1992). Machine learning and artificial intelligence: an introduction. Analytical chemistry (Washington, DC), 64(1), 49-60 https://doi.org/10.1021/ac00025a742

[41] Courbariaux, M., Hubara, I., Soudry, D., El-Yaniv, R., \& Bengio, Y. (2016). Binarized neural networks: Training deep neural networks with weights and activations constrained to +1 or -1, Computer Science, Machine Learning, Cornell University, 1-13. Arxiv:1602.02830.

[42] Zhu, B., Al-Ars, Z., \& Pan, W. (2020). Towards lossless binary convolutional neural networks using piecewise approximation, Computer Science, Machine Learning, Cornell University, 1-8. ArXiv:2008.03520.

[43] Yadav, S. \& Shukla, S. (2016). Analysis of k-fold crossvalidation over hold-out validation on colossal datasets for quality classification. 2016 IEEE 6th International conference on advanced computing (IACC), 78-83. https://doi.org/10.1109/IACC.2016.25

[44] Desmoulin, G. T. \& Anderson, G. S. (2011). Method to investigate contusion mechanics in living humans. Journal of Forensic Biomech, 2 1-10. https://doi.org/10.4303/ffb/F100402

[45] Otamendi, G. S. V. (2011). Designing deformable models of soft tissue for virtual surgery Planning and simulation using the Mass-Spring Model. School of Engineering, University of Navarra, Spain

[46] Rebello, K. J. (2004). Applications of MEMs in surgery.' Proceedings of the IEEE, 92(1), 43-55. https://doi.org/10.1109/JPROC.2003.820536

[47] Varady, P., Nagy, L., \& Szilagyi, L. (2000). On-line detection of sleep apnea during critical care monitoring, InProceedings of the22nd Annual International Conference of the IEEE Engineering in Medicine and Biology Society, 2, 1299-1301. https://doi.org/10.1109//EMBS.2000.897976

[48] Rendón, D. B., Ojeda, J. L. R., Foix, L. F. C., Morillo, D. S., \& Fernández, M. A. (2007). Mapping the human body for vibrations using an accelerometer. 29th Annual International Conference of the IEEE Engineering in Medicine and Biology Society, 1671-1674. https://doi.org/10.1109/IEMBS.2007.4352629 


\section{Contact information:}

Okan ORAL, Assist. Prof. Dr.

(Corresponding author)

Akdeniz University,

Department of Mechatronics Engineering,

Engineering Faculty Dumlupinar Boulevard 07058 Campus Antalya Turkey

E-mail: okan@akdeniz.edu.tr

Suleymn BILGIN, Assoc. Prof. Dr.

Akdeniz University,

Department of Electric and Electronics Engineering,

Engineering Faculty Dumlupinar Boulevard 07058 Campus Antalya Turkey

E-mail: sbilgin@akdeniz.edu.tr

Mehmet Umit AK, PhD Student

Akdeniz University,

Department of Electric and Electronics Engineering,

Engineering Faculty Dumlupinar Boulevard 07058 Campus Antalya Turkey

E-mail: mehmetumitak@akdeniz.edu.tr 